Characterization of Medipix3 with the MARS readout and software

This article has been downloaded from IOPscience. Please scroll down to see the full text article.

2011 JINST 6 C01056

(http://iopscience.iop.org/1748-0221/6/01/C01056)

View the table of contents for this issue, or go to the journal homepage for more

Download details:

IP Address: 137.138.124.233

The article was downloaded on 27/07/2012 at 08:24

Please note that terms and conditions apply. 


\title{
Characterization of Medipix3 with the MARS readout and software
}

\author{
J.P. Ronaldson, ${ }^{a, 1}$ M. Walsh, ${ }^{a}$ S.J. Nik, ${ }^{b}$ J. Donaldson, ${ }^{b}$ R.M.N. Doesburg, ${ }^{b}$ \\ D. van Leeuwen, ${ }^{c}$ R. Ballabriga, ${ }^{d}$ M.N. Clyne, ${ }^{e}$ A.P.H. Butler ${ }^{a, c, d}$ and P.H. Butler ${ }^{a, d}$ \\ ${ }^{a}$ Department of Radiology, University of Otago, \\ Christchurch 8140, New Zealand \\ ${ }^{b}$ Department of Physics and Astronomy, University of Canterbury, \\ Christchurch 8140, New Zealand \\ ${ }^{c}$ Department of Electrical and Computer Engineering, University of Canterbury, \\ Christchurch 8140, New Zealand \\ ${ }^{d}$ European Organization for Nuclear Research \\ Geneva 23, Switzerland \\ eI.L.R Ltd., \\ Christchurch 8025, New Zealand \\ E-mail: ronjo871@student .otago.ac.nz
}

ABSTRACT: The Medipix3 x-ray imaging detector has been characterized using the MARS camera. This x-ray camera comprises custom built readout electronics and software libraries designed for the Medipix family of detectors. The performance of the Medipix3 and MARS camera system is being studied prior to use in real-world applications such as the recently developed MARSCT3 spectroscopic micro-CT scanner. We present the results of characterization measurements, describe methods for optimizing performance and give examples of spectroscopic images acquired with Medipix3 and the MARS camera system.

A limited number of operating modes of the Medipix 3 chip have been characterized and singlepixel mode has been found to give acceptable performance in terms of energy response, image quality and stability over time. Spectroscopic performance is significantly better in charge-summing mode than single-pixel mode however image quality and stability over time are compromised. There are more modes of operation to be tested and further work is required to optimize the performance of the chip.

KeYwords: X-ray detectors; Spectrometers; Computerized Tomography (CT) and Computed Radiography (CR)

\footnotetext{
${ }^{1}$ Corresponding author.
} 


\section{Contents}

1 Introduction 1

2 Methods and materials 2

2.1 Equipment 2

2.2 Threshold equalization 2

2.3 Counting stability 3

2.4 Spectroscopy 3

3 Results 4

3.1 Threshold equalization 4

3.2 Counting stability 4

3.3 Spectroscopy 6

4 Discussion $\quad 8$

5 Conclusions $\quad 8$

\section{Introduction}

The Medipix family of x-ray imaging detectors are developed by an international collaboration led by designers at CERN. The collaboration aims to apply detector technology developed in support of high energy physics to medical imaging. Medipix 3 is the latest and most advanced in this series of photon counting detectors and is currently undergoing testing and evaluation.

The Medipix 3 [1,2] extends the capabilities of earlier detectors by including additional operating modes and configuration options. Charge-summing mode is designed to mitigate the unwanted charge-sharing effects that degrade the spectroscopic performance of fine-pitch pixel counters such as Medipix2 [3]. A system of pixel summing and arbitration circuits is provided to sum the charge over adjacent pixels and allocate the result to the pixel contributing the largest charge. Medipix 3 also provides multiple readout counters and discriminator thresholds per pixel. In fine pitch mode the $55 \mu \mathrm{m} \times 55 \mu \mathrm{m}$ sensor layer and readout layer pixels are arranged in a one-to-one mapping with two readout counters per detector pixel. In spectroscopic mode, four Medipix3 readout pixels are mapped to one $110 \mu \mathrm{m} \times 110 \mu \mathrm{m}$ sensor pixel thereby providing eight readout counters and discriminator thresholds per pixel.

The performance of the MARS-Medipix 3 camera system has been characterized in singlepixel and charge-summing modes. We present the results of characterization measurements, describe methods for optimizing performance and give examples of spectroscopic images acquired with Medipix3 and the MARS camera system. 

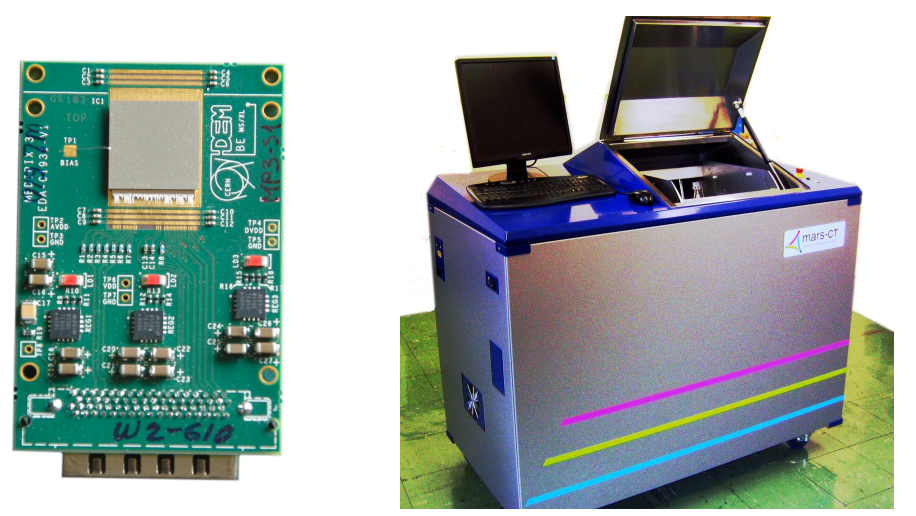

Figure 1. The Medipix3 detector with $300 \mu \mathrm{m}$ thick silicon sensor layer and the MARS-CT3 system incorporating the MARS camera and Source-Ray SB-80-1K x-ray tube.

\section{Methods and materials}

\subsection{Equipment}

A $256 \times 256$ pixel Medipix 3 bump-bonded to a $300 \mu \mathrm{m}$ thick silicon sensor layer was used for this work (see figure 1). It was connected to the MARS camera readout via an external SCSI cable and cooled by ambient air only. The prototype MARS camera was controlled by the locally developed libMars C library and Python interface. For all measurements the Medipix3 was operated in high gain mode.

For the characterization measurements the MARS camera unit was used in standalone mode together with a Kevex PSX11-150-75 mini-focus X-ray tube with tungsten anode and $0.13 \mathrm{~mm}$ beryllium window. For the projection scanning measurements, the detector and samples were mounted within the MARS-CT3 micro-CT system. The MARS-CT3 was equipped with a SourceRay SB-80-1K bremsstrahlung $\mathrm{x}$-ray tube with tungsten anode and $1.8 \mathrm{~mm}$ of aluminium (equivalent) intrinsic filtration.

\subsection{Threshold equalization}

Due to intrinsic variations in the threshold levels associated with each counter, the responses of the individual pixels to a uniform flux are not consistent across the chip. Before acceptable images can be acquired it is necessary to equalize the individual discriminator threshold levels so that each is sensitive to pulses of the same minimum height. Two global current sources (one positive and one negative) and 5 adjustment bits per individual discriminator are available for this purpose. The negative current source is set by a global DAC and may be applied at the pixel level to any discriminator threshold using a switch selected by one of the 5 adjustment bits. Similarly the positive current source is set by another global DAC and some fraction of this may be applied to each discriminator threshold using the remaining 4 adjustment bits.

The equalization process applied to the MARS camera used the intrinsic noise of the chip to find optimum threshold configuration masks for single-pixel mode (SPM) and charge-summing mode (CSM). Prior to equalization in SPM all pixels were switched on and a null adjustment mask applied. An initial threshold scan was used to set the negative current selection bit for those pixels 
with discriminator thresholds above the noise floor. Subsequent scans were used to iteratively adjust the positive current selection bits to equalize the position of the discriminator thresholds with respect to the noise floor for each pixel. Any remaining noisy pixels were identified as outliers and their operation inhibited. The equalization procedure for CSM was similar except that equalization was performed sequentially for four sets of pixels - each set comprising corresponding members of local clusters of $2 \times 2$ pixels. The remaining $75 \%$ of pixels were switched off during each part of the process to eliminate arbitration effects. The four parts of the CSM configuration mask were then combined.

The effectiveness of the equalization process was examined by measuring the normalized flatfield responses (gain map) in SPM and CSM. A successful equalization should yield a gain map that is approximately uniform across all pixels. This was tested in SPM and CSM by illuminating the chip with a $50 \mathrm{kVp}$ flat-field. The pixel responses were averaged over one hundred frames and normalized. The distribution of pixel gain values was then examined graphically.

\subsection{Counting stability}

The ability of the chip to acquire repeatable measurements depends upon the stability of the bias signals supplied by the global DACs. The libMars Python interface was used to monitor the output of the global DACs during a continuous series of threshold scans of the intrinsic noise in SPM and CSM. The observed output levels were plotted for a period of approximately 40 minutes after power-on from a cold state and examined for unwanted variations.

For a stable detector the counts observed during repeated measurements under identical conditions are expected to follow a Poisson distribution. This was investigated with the detector in SPM exposed to a flat-field $\mathrm{x}$-ray source. A set of repeated measurements were taken and the sample variance to mean ratio calculated. This dispersion index is expected to follow a $\chi^{2}$ distribution with $N-1$ degrees of freedom where $N$ is the number of samples. The observed results were compared graphically with the theoretical distribution.

\subsection{Spectroscopy}

The energy response of the MARS-Medipix 3 camera system was calibrated using threshold scans of $x$-ray fluorescence spectra generated from indium and molybdenum samples placed within the $50 \mathrm{kVp} \mathrm{x}$-ray beam. The global (sum over all pixels) detector response resolution was estimated from the width of the molybdenum $\mathrm{K}_{\alpha}$ fluorescence peak at $17.5 \mathrm{keV}$.

To validate the performance of the Medipix 3 a small number $(n)$ of spectroscopic imaging measurements were made of a high contrast test object (a USB flash disk) and a biological sample (a preserved mouse specimen injected with iodine and barium contrast media). The image data were used to form a set of spectroscopic vectors (one $n$-vector per pixel). The spectroscopic pixel data set was analysed and categorized using a self-organising map (SOM). ${ }^{1}$ A colour was assigned to each pixel category and the corresponding pixels labelled accordingly to create an artificial colour-x-ray image. The image was examined to confirm that the pixels had been correctly clustered according to their expected spectroscopic properties.

\footnotetext{
${ }^{1}$ The SOM, also known as a Kohonen map [4], is an unsupervised learning algorithm akin to a neural network which in this case was configured to categorize pixels according to similarities in their spectroscopic vectors.
} 

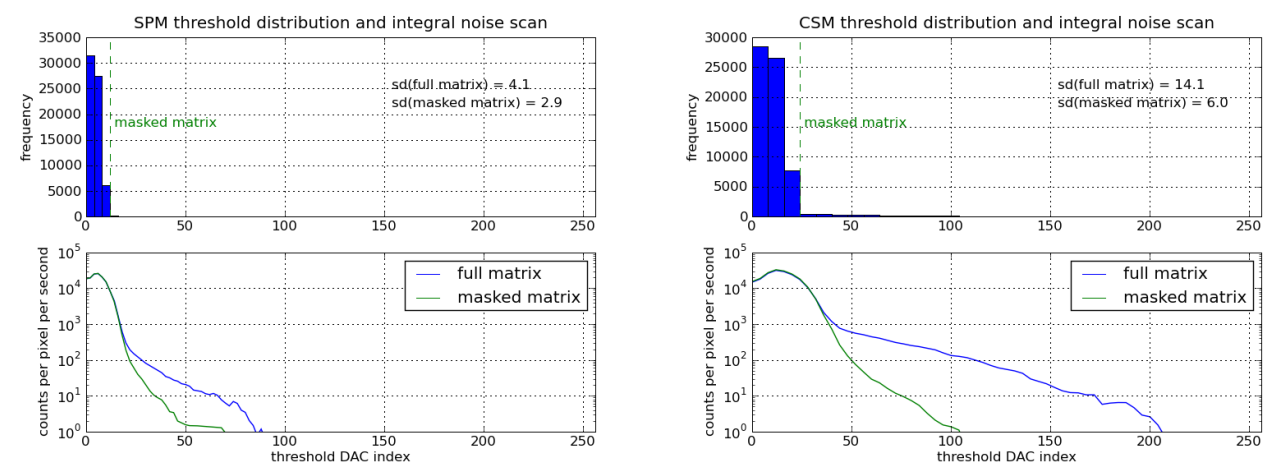

Figure 2. Discriminator threshold distributions and integral noise scans in SPM and CSM. In SPM the threshold noise scan taken with the full pixel matrix is compared with that obtained when pixels with a threshold $>12$ DAC steps are masked. In CSM the full pixel matrix noise scan is compared with the scan obtained when pixels with a threshold location $>24$ DAC steps are masked.

\section{Results}

\subsection{Threshold equalization}

Figure 2 shows the discriminator threshold distributions and integral noise scans after equalization in SPM and CSM. After decreasing rapidly with increasing threshold to $\sim 20$ DAC steps, the integral noise scan using the full pixel matrix diminished only slowly over the range from $\sim 20-100$ DAC steps. Masking the pixel matrix to remove those pixels with a threshold $>12$ DAC steps produced a more compact noise scan. The residual threshold dispersion of the masked pixel matrix was $\sigma \simeq 3$ DAC steps ( $\equiv 420 \mathrm{eV} \equiv 120 \mathrm{e}^{-}$at current settings). A similar pattern is observed after equalization in CSM except that the threshold distribution and noise scans were approximately twice as wide. The residual dispersion of the masked matrix in CSM was $\sigma \simeq 6$ DAC steps ( $\equiv 840 \mathrm{eV} \equiv 230 \mathrm{e}^{-}$at current settings).

The SPM gain map (figure 3) was approximately normally distributed and tightly clustered about unity. However the CSM gain map distribution was widely dispersed and strongly skewed. These results are reflected in the test images of a USB flash disk (figure 4). The SPM test image shows little evidence of fixed pattern noise unlike the CSM test image which is of poor quality and shows a high degree of bias towards certain pixels.

\subsection{Counting stability}

A number of DAC outputs showed evidence of unwanted variation (figure 5). The outputs from the Cas and FBK DACs showed the most significant variation and this was greatest in the period immediately after power-on. In SPM the DAC outputs stabilized after a few minutes. In CSM although the rate of variation quickly reduced, the Cas and FBK output levels continued to change throughout the monitoring interval ( $\sim 40$ minutes).

Figure 6 shows the dispersion index (variance/mean) distribution for repeated measurements under identical conditions. For the series of SPM measurements the observed dispersion index distribution was in good agreement with the theoretical $\chi^{2}$ distribution expected from Poisson counting statistics. 

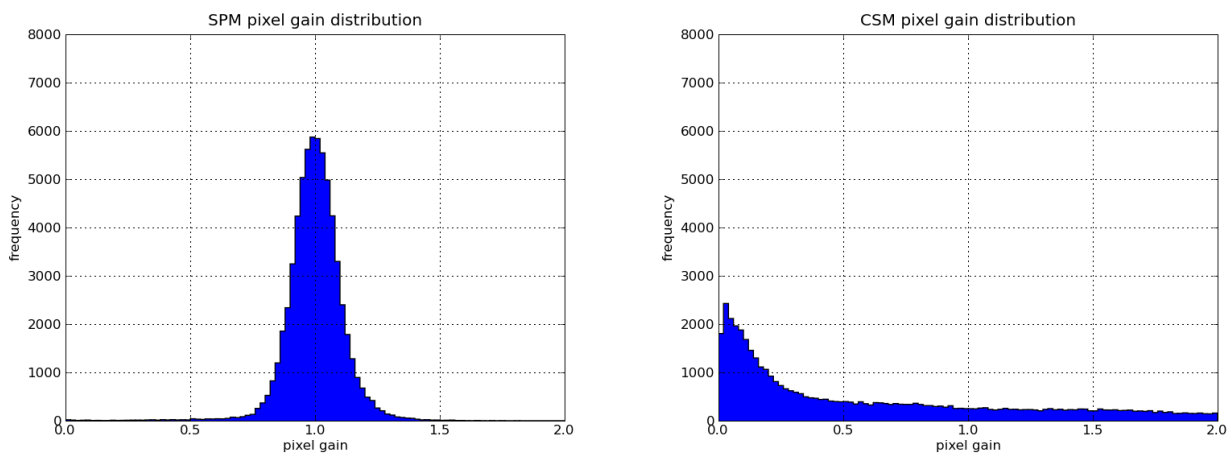

Figure 3. Distributions of relative count rates (pixel gain values) for a flat-field $x$-ray source measured in SPM and CSM. $(V=50 \mathrm{kVp} ; E>12 \mathrm{keV}$.)
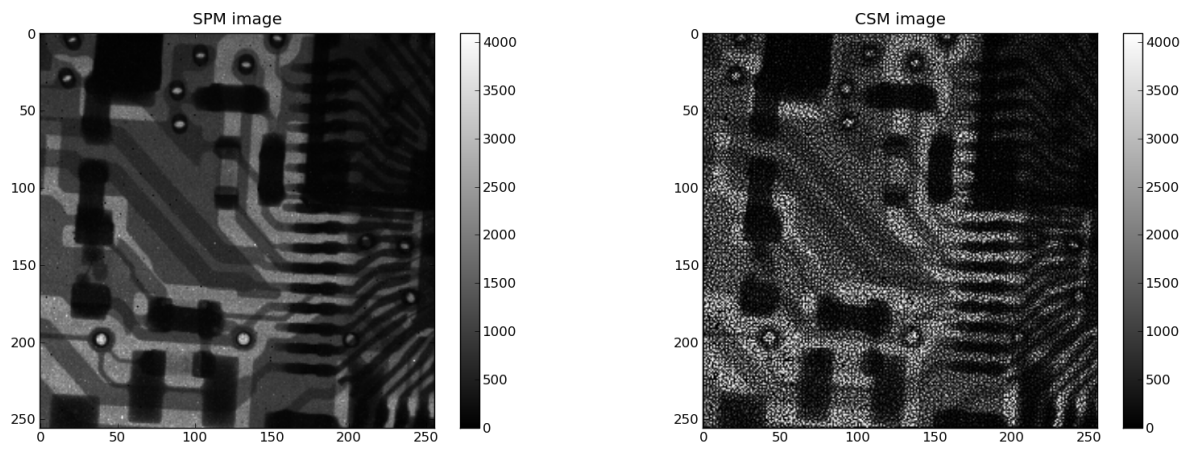

Figure 4. Test images of a USB flash-disk in SPM and CSM. $(V=50 \mathrm{kVp} ; E>12 \mathrm{keV}$.)
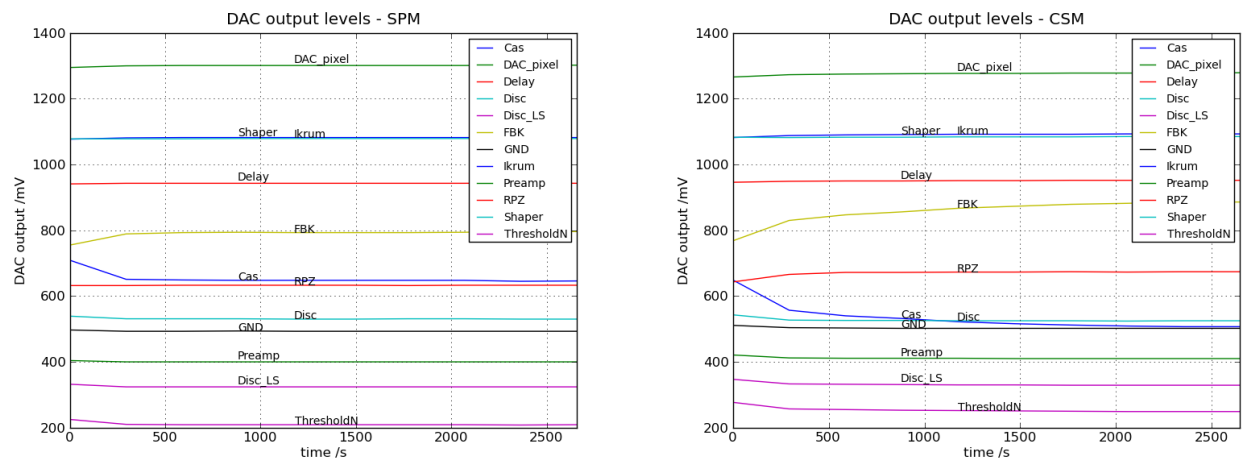

Figure 5. The DAC outputs of the Medipix3 recorded during continuous threshold scans of the intrinsic noise initiated immediately after power-on. 


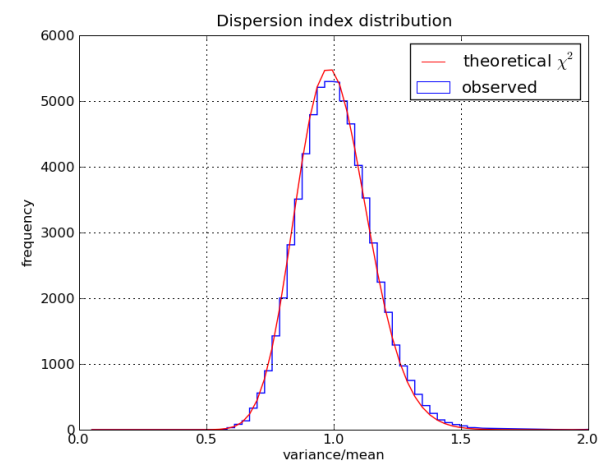

Figure 6. The dispersion index (variance/mean) distribution calculated from repeated SPM measurements and the theoretical $\chi^{2}$ distribution expected from Poisson counting statistics. $(V=50 \mathrm{kVp} ; E>12 \mathrm{keV}$.)

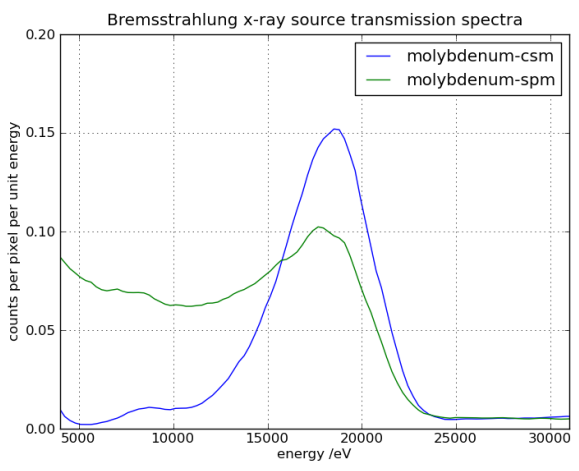

Figure 7. SPM and CSM spectra from fluorescence $\mathrm{x}$-rays generated in a $100 \mu \mathrm{m}$ molybdenum filter placed within the beam of the $\mathrm{x}$-ray tube. $(V=50 \mathrm{kVp}$. $)$

\subsection{Spectroscopy}

The SPM and CSM pulse height spectra for fluorescence $\mathrm{x}$-rays generated in a molybdenum filter placed directly within the beam of the x-ray tube are shown in figure 7. The application of CSM improved the quality of the spectra by reducing the low energy continua caused by the charge-sharing effects. The global (sum over all pixels) FWHM of the molybdenum fluorescence peak in CSM was $\simeq 5 \mathrm{keV}$.

The results of spectroscopic imaging of the USB flash disk and analysis by SOM are shown in figure 8. Pixels corresponding to similar material types have been correctly clustered into similar categories. In particular the solder material (containing tin and lead) is easily distinguished from the lighter copper of the electronic tracks and the plastic material used in the body of the device.

Figure 9 shows the results of similar measurements and analysis of the mouse specimen. In this case the SOM has correctly distinguished various types of biological materials and artificial contrast media according to their spectroscopic properties. 


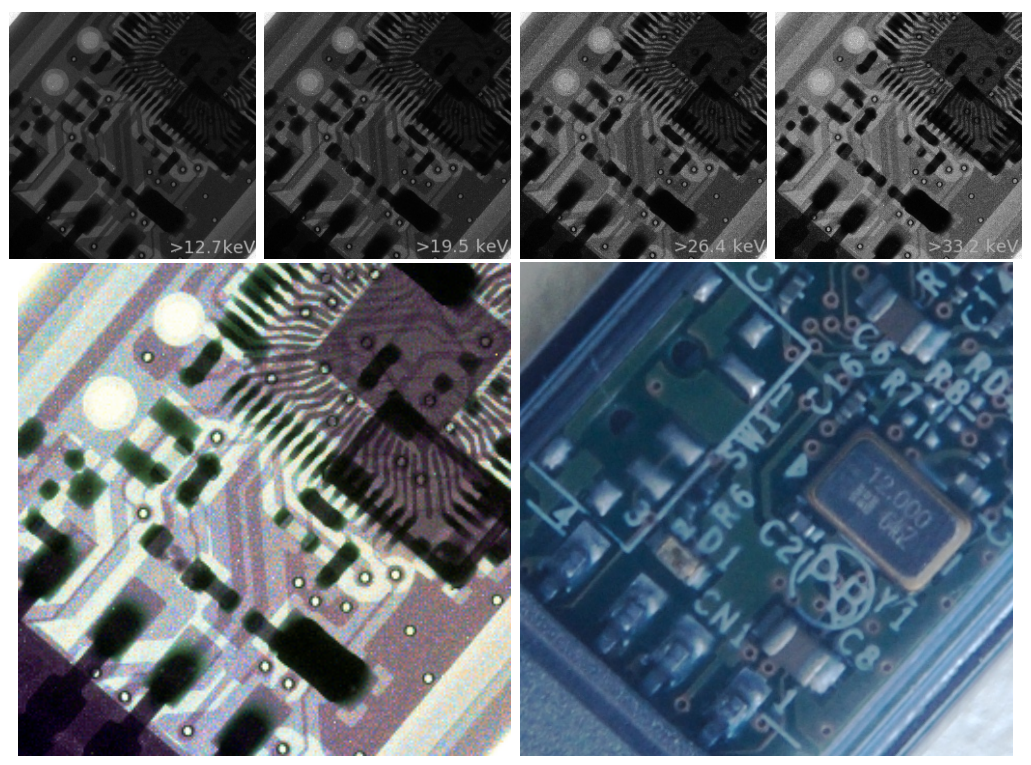

Figure 8. Top: A series of spectroscopic transmission images of a USB flash disk acquired with four different detector energy thresholds using a fixed x-ray tube voltage of $50 \mathrm{kVp}$. Bottom: An enhanced colour $\mathrm{x}$-ray image derived from the individual spectroscopic images compared with a photograph of the USB flash disk. $(V=50 \mathrm{kVp} ; E>12.7,>19.5,>26.4,>33.2 \mathrm{keV}$. $)$

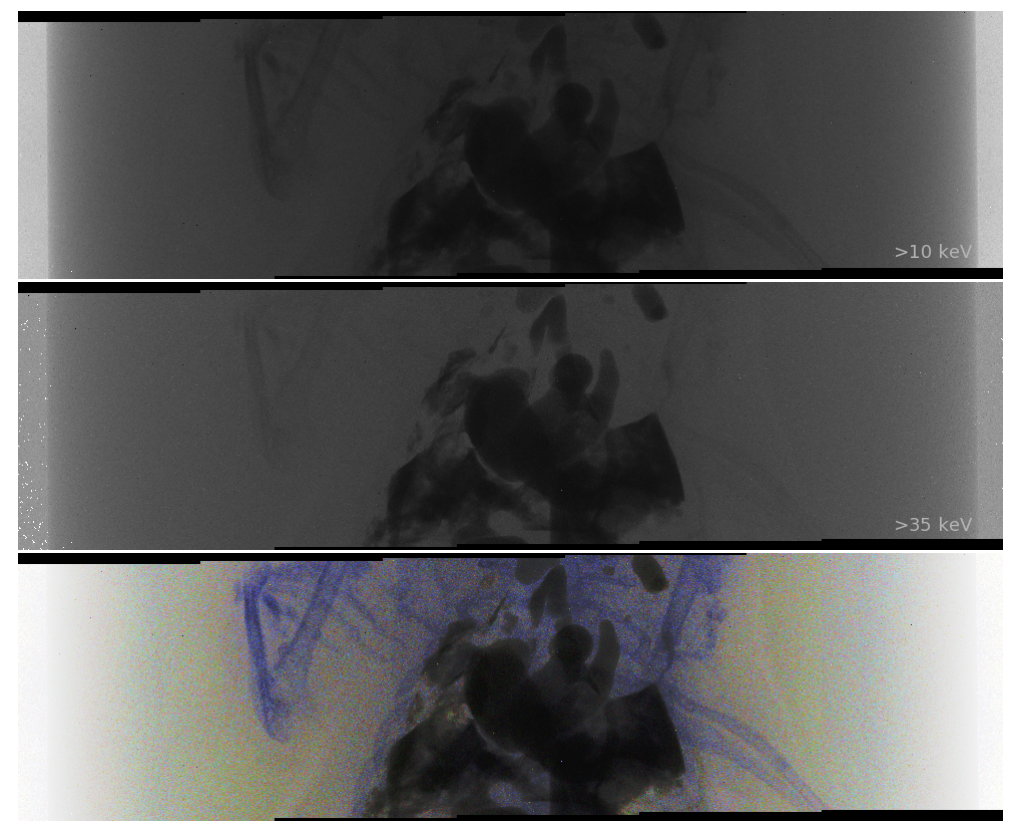

Figure 9. Two spectroscopic transmission images of the abdominal region of a preserved mouse containing artificial contrast media and an enhanced colour x-ray image derived from the same data. $(V=80 \mathrm{kVp} ; E>10,>35 \mathrm{keV}$.) 


\section{Discussion}

For this work acceptable performance in SPM was achieved by allowing sufficient time for stabilization within the data acquisition protocols. However the failure of the CSM DAC levels to stabilize within the monitoring interval currently prevents the use of this mode in routine scanning operations. The analogue power consumption in CSM $(15.45 \mu \mathrm{W}$ per pixel) is higher than in SPM $(8.25 \mu \mathrm{W}$ per pixel) and the variation in DAC output levels may be related to the increased heat output. The MARS camera uses an external DAC to provide the Cas signal and attempts to stabilize this and the internal Medipix3 FBK DAC on startup using a software controlled feedback loop. Considering the proposed MARS-CT3 micro-CT scanner applications [5], the enclosed installation environment and high radiation fields in which the chip will operate may further exacerbate the heating effect. If the current stabilization methods and cooling with ambient air are insufficient then an alternative means of stabilizing the chip temperature such as Peltier cooling will be required.

The global energy resolution of the detector is a function of quantum counting effects, random electronic noise and systematic effects such as residual threshold dispersion. In room temperature silicon, the contribution of quantum counting effects is relatively minor and we expect random electronic noise and threshold dispersion to dominate. After equalization the threshold dispersion is fixed at the pixel level. In CSM four single-pixel channels are summed to provide each output. The threshold dispersion and electronic noise distributions add in quadrature giving a total CSM pulse height distribution that is approximately twice the width of that in SPM. Our measured value for residual threshold dispersion $(\sigma \simeq 840 \mathrm{eV})$ implies a lower limit on the achievable CSM energy resolution of FWHM $>\sim 2 \mathrm{keV}$.

The SPM spectroscopic imaging results successfully demonstrate the use of the chip for biological materials discrimination. The mouse sample was scanned sequentially using only two distinct energy thresholds but it was nevertheless possible to distinguish various material types using the spectroscopic information. The benefits and optimum configuration for simultaneous acquisition into the two counters per pixel available with Medipix 3 in fine pitch mode and eight counters per pixel in spectroscopic mode remain to be explored.

\section{Conclusions}

The performance of the MARS-Medipix3 camera combination has been characterized and, with some limitations, shown to be acceptable for spectroscopic imaging. Single-pixel mode has been found to give acceptable performance in terms of energy response, image quality and stability over time. Installation of the MARS-Medipix 3 camera within the MARS-CT3 for use as a spectroscopic $\mathrm{x}$-ray imaging research tool is therefore a feasible option. The effects of instabilities in DAC output have been minimised in SPM through appropriate measurement protocols. However more robust methods of control such as Peltier cooling may be appropriate for future applications.

\section{Acknowledgments}

The authors would like to thank all the members of the Medipix2 and Medipix 3 collaborations, the MARS-CT research team, and the Canterbury District Health Board for their valuable support. 


\section{References}

[1] R. Ballabriga, M. Campbell, E. Heijne, X. Llopart and L. Tlustos, The Medipix3 prototype, a pixel readout chip working in single photon counting mode with improved spectrometric performance, IEEE Nucl. Sci. Conf. R. 6 (2006) 3557.

[2] R. Ballabriga, M. Campbell, E. Heijne, X. Llopart, L. Tlustos and W. Wong, Medipix3: A 64k pixel detector readout chip working in single photon counting mode with improved spectrometric performance, Nucl. Instrum. Meth. A in press, corrected proof (2010).

[3] X. Llopart, M. Campbell, R. Dinapoli, D. San Segundo and E. Pernigotti, Medipix2: A 64 k pixel readout chip with 55 micron square elements working in single photon counting mode, IEEE Trans. Nucl. Sci. 49 (2002) 2279.

[4] T. Kohonen, The self-organizing map, Proc. IEEE 78 (1990) 1464.

[5] M.F. Walsh et al., Spectral CT using Medipix3 and the MARS-CT3 scanner, Manuscript submitted for publication, (2010). 